\title{
Solitary spinal osteochondroma causing neural syndromes
}

\author{
A Prasad MD, P N Renjen MD, M L Prasad MD, G B Bhatty MD, V S Madan MD. \\ T B S Buxi MD, S P Agarwal MD
}

Department of Neurosurgery, Neurology, Pathology and Radiology, Sir Ganga Ram and RML Hospitals, New Delhi, India.

\begin{abstract}
Three cases of solitary osteochondroma of the spine are reported. Two presented with cervical myelopathy and one with radiculopathy. Plain radiography or tomography is adequate for the diagnosis in the majority of cases; nevertheless, a CT scan or MRI is of immense help in planning surgical treatment. The radiological features of spinal exostoses are reviewed.
\end{abstract}

Key words: spinal osteochondroma; cervical myelopathy; cervical radiculopathy.

\section{Case reports}

\section{Case 1}

A 16 year old girl was admitted in January 1991 for evaluation of progressive weakness and numbness of the lower limbs of 6 months duration. On examination moderate spastic paraparesis was noted. Sensations were partially impaired below the L3 dermatome. Plain radiographs of the thoracic spine showed a well circumscribed bony mass arising from the right pedicle of T10. Myelography demonstrated a partial extradural block at the T10 vertebral body with a shift of contrast to the left. CT scan clearly delineated the bony component of an extradural exostosis arising from the right pedicle of T10.

At operation a large bony mass was noted to arise from the right T10 pedicle displacing the spinal cord to the left. Piecemeal total decompression of the mass was performed. Histopathological examination revealed a centre of mature bone with normal hemopoietic tissue in between the lamellar trabeculae. A cartilaginous cap was present at the periphery which was separated from the centre by a zone of endochondral ossification. Neurological examination one year postoperatively revealed no neurological deficit.

\section{Case 2}

A 20 year old man was admitted in July 1990 for the evaluation of progressive weakness and wasting of the left upper limb of 6 months duration. Neurological examination revealed

Correspondence: B-60, New Rajinder Nagar, New Delhi 110060, India. global wasting of the left upper limb and a mild left-sided hemiparesis. Sensations were grossly impaired on the left side of the body. A tomogram of the cervical spine showed a well circumscribed bony projection, arising from the spinous process and the left lamina of $\mathrm{C} 2$, projecting superiorly into the spinal canal just ventral to the posterior arch of C1. Myelography revealed a large filling defect in the contrast column which was larger than the bony projection. A myelo-CT scan only revealed the bony portion of the exostosis (Fig 1).

At operation, the posterior arch of $\mathrm{Cl}$ and $\mathrm{C} 2$ spines and laminae were defined. The laminae of $\mathrm{C} 2$ were nibbled on either sides of the exostosis. Two blunt broad dissectors were used to maintain retraction over the exostosis and to avoid aggravation of the spinal cord compression. Minor adhesions between the exostosis and the spinal cord were removed with the help

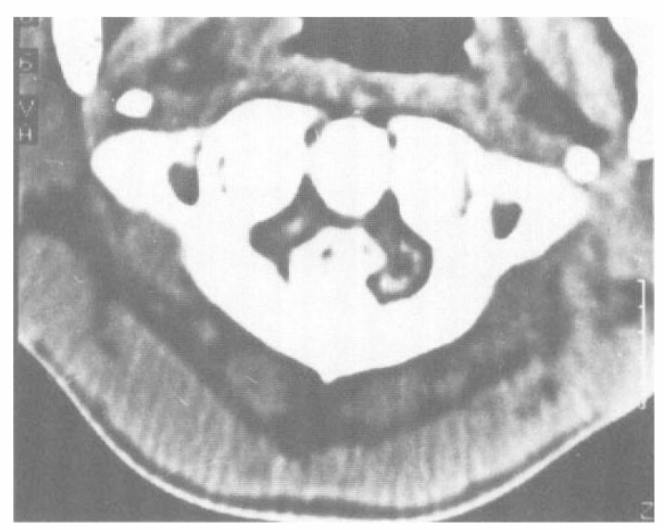

Figure 1 Myelo-CT scan showing an extradural exostosis arising from the spine of $\mathrm{C} 2$. 
of a fine dissector, and the exostosis was removed as a single mass. Microscopic examination revealed the characteristic findings of an exostosis. One and a half year later the patient was asymptomatic, but neurological examination showed remarkable wasting of the left upper limb.

\section{Case 3}

A 60 year old man presented in June 1990 with 3 episodes of intermittent moderate degree pain in the right nape of neck for 2 months lasting for a few days; one of these episodes clearly related to flexion of the neck during exercise. Neurological examination was normal. Plain radiographs of the cervical spine revealed a well circumscribed mound-shaped bony mass arising from the right lamina and pedicle of C3 (Fig 2). The patient refused to have a myelo-CT scan of the cervical spine. He was advised to avoid extremes of neck movement. During the one and a half year follow up he has had a few episodes of intermittent mild neck pain. Repeat radiographs at this time did not reveal any increase in the size of the exostosis.

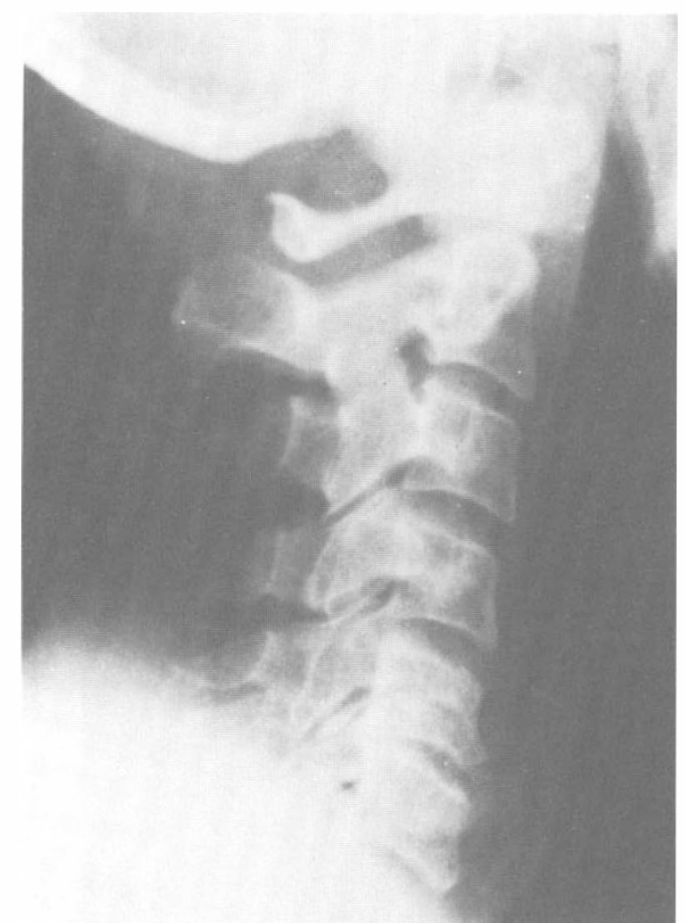

Figure 2 Plain radiograph showing a well circumscribed mound-shaped bony mass arising from the lamina of $\mathrm{C} 3$.

\section{Discussion}

A spinal exostosis (osteochondroma) is a protrusion of a well circumscribed moundshaped mass from the neural arch. The protrusion consists of a pedunculated or sessile bony stalk covered with a cartilaginous cap. The surface of the cap is rounded, mushroom-like or irregularly bosselated. The cortical and intramedullary trabecular pattern of the host bone blends with that of the exostosis. No other lesion demonstrates this flaring. ${ }^{1}$ About $80 \%$ of these lesions arise from long bone, especially about the knee and in the upper humerus. The pelvis, scapula and ribs are involved in about $12 \%$ of cases. Not more than $2 \%$ of these lesions are located in the spine. The exostosis increases in size during childhood and adolescence by endochondral ossification. Spinal cord compression due to a solitary or to multiple exostoses is rare. ${ }^{2,3}$ An exostosis producing neurological symptoms in a patient beyond 40 years of age, as seen in case 3 , is rarer. Narrowing of the spinal canal due to degenerative changes, rather than the growth of the exostosis, may explain the delayed appearance of neurological symptoms in this age group of patients. The medical history was remarkable for the relationship between the neurological symptoms and neck flexion in 2 patients of the present series. Increase of upper cervical pain with rotation of the head has been noted earlier in a case of $\mathrm{C} 1$ exostosis. ${ }^{4}$ When the neck is flexed the posterior dura is drawn tight producing pressure over the spinal cord and nerve roots. This may account for the increase of symptoms in patients with an exostosis on neck flexion.

Plain radiographs may show the bony projection of the exostosis, but this will not outline the cartilaginous cap. However, it may be unremarkable when the mineralisation of the bony part is inadequate. Tomography shows the bony projection arising from the neural arch unequivocally. The characteristic appearance of an exostosis is usually that of a calcified, sessile or pedunculated, round or mound shaped mass. Myelography reveals a filling defect in the contrast column which is larger than the bony projection, as was shown in case 2 . CT scan demonstrates its relationship to the 
paraspinal and intraspinal tissues very well. However, it fails to show the cartilaginous cap in the majority of cases. ${ }^{5}$ In the present series, the CT scan did not show the cartilaginous cap in either of the cases in which a CT scan was performed. Nevertheless, it did demonstrate the thicker cartilage (usually more than $2 \mathrm{~cm}$ ) in 14 of the 15 exostosis chondrosarcomas, although the measurements of the maximum thickness were often imprecise. ${ }^{5}$

MRI studies of an exostosis show well defined bone, cartilage and marrow signal intensities and the entire lesion, including the cartilaginous cap, is clearly delineated in T2 dependent images. The majority of authors feel that if the maximum thickness of the cartilaginous cap exceeds $2 \mathrm{~cm}$, a sarcomatous transformation must be seriously considered. ${ }^{1}$ Since the surgical treatment of a benign exostosis and exostotic chondrosarcoma varies, a preoperative diagnosis is essential for their management. In the 2 cases of spinal exostosis in which MRI was performed, intramedullary abnormalities were not demonstrated. ${ }^{3,6}$
Chondrosarcomatous changes have been observed in $<1 \%$ of cases of solitary exostosis; whereas in cases of hereditary multiple exostosis it is seen with greater frequency. Thus the authors feel that MRI may not be very useful in cases of solitary exostosis. However it may prove to be helpful (1) when the radiological diagnosis is in doubt; and (2) when sarcomatous degeneration is suspected in patients with hereditary multiple exostoses.

An exostosis arising from the lamina and pedicle may be removed by a posterior approach. However, a mass occupying the central part of the vertebral body has to be excised by an anterior approach. A mass arising from the lamina may be removed as a single mass, as was done in case 2 of the present series; whereas that from the pedicle or the body should be removed in a piecemeal fashion. At operation, any manoeuvre which might increase spinal cord compression, such as a rocking movement of the exostosis, and the insertion of a rongeur beneath the lamina must be avoided.

\section{References}

1 Mirra JM (1989) Parosteal tumors. In: Mirra JM, editor. Bone Tumors: Clinical, Radiologic, and Pathologic Correlations. Lea and Febiger, Philadelphia: 1627-1753.

2 Spaziante R, Irace C, Gambardella A, Cappabianca P, De Divitiis E (1988) Solitary osteochondroma of the pedicle of L4 causing root compression. J Neurosurg Sci 32: 141-145.

3 Moriwaka F, Hozen H, Nakane K, Sasaki H, Tashiro K, Abe H (1990) Myelopathy due to osteochondroma: MR and CT studies. J Comput Assist Tomogr 14: 128-130.

4 Julien J, Riemens V, Vital CI, Lagueny A (1978) Cervical cord compression by solitary osteochondroma of the atlas. J Neurol Neurosurg Psychiatry 41: 479-481.

5 Hudson TM, Springfield DS, Spanier SS, Enneking WF, Hamlin DJ (1984) Benign exostosis and exostotic chondrosarcomas: Evaluation of cartilage thickness. Radiology 152: 595-599.

6 Tully RJ, Pickens J, Oro J, Levine C (1989) Hereditary multiple exostoses and cervical cord compression: CT and MR studies. J Comput Assist Tomogr 13: 330-333. 